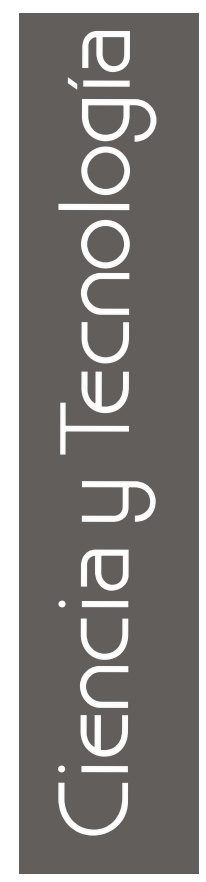

\title{
Efecto del Escaldado sobre la Deshidratación Osmótica del Mango
}

* Profesores de la Universidad Politécnica de Valencia España.

** Estudiante de Pregrado - Universidad Politécnica de Valencia - España.

*** Ph.D. Profesor Asistente - Departamento de Ingeniería de Alimentos - Facultad de Ingeniería - Universidad del Valle Santiago de Cali.

E-mail:alfayala@univalle.edu.co

Fecha de recepción: Moviembre 15 de 2002

Fecha de aprobación: Marzo 26 de 2003
Javier Martinez*

Ana Calero**

Alfredo Ayala Aponte***

Amparo Chiralt*

Pedro Fito*.

\section{RESUMEN}

Los tratamientos combinados como el escaldado, deshidratación osmótica a presión atmosférica (OD) y deshidratación osmótica con pulso de vacío (PVOD) se emplean en el procesamiento de frutas para mejorar su calidad (sensorial y nutricional). El escaldado reduce la actividad enzimática y en algunos casos mejora el color en las frutas. La reducción de la actividad de agua mediante el proceso de deshidratación osmótica con la aplicación de disoluciones concentradas de sal o azúcar, a baja temperatura, permite reducir el contenido de agua congelable de frutas procesadas mínimamente, para mejorar su conservación en congelación. En este trabajo 
se analizó la influencia del pretratamiento del escaldado de mango (var. Kent) sobre la cinética de deshidratación osmótica a presión atmosférica y con pulso de vacío. Los tratamientos se realizaron a $30^{\circ} \mathrm{C}$ con una disolución de sacarosa concentrada a 59.3 Brix. Para cada tiempo del proceso (hasta 5 h) las muestras se analizaron en su variación de peso y volumen, contenido de humedad, concentración de sólidos solubles y actividad de agua. La cinética de las ganancias netas de soluto y agua se analizaron en términos de la composición de la fase líquida de la fruta (FLM: agua más sólidos solubles). Los tratamientos PVOD mostraron cinéticas más rápidas en ganancias de soluto y pérdida de agua, y valores ligeramente más altos de difusividad efectiva $\left(D_{e}\right)$ en la FLM, especialmente en los pretratamientos de escaldado. En los tratamientos OD los cambios de volumen fueron explicados en términos de la FLM. Los tratamientos PVOD presentaron una desviación de la variación de volumen frente a OD debido a la impregnación deformación durante el pulso de vacío.

Palabras clave: Deshidratación osmótica, escaldado, mango.

\section{ABSTRACT}

Combined treatments, such as blanching, osmotic dehydration at atmospheric pressure (OD) and pulsed vacuum osmotic dehydration (PVOD) to improve food quality (sensorial and nutritional) are a useful practice in fruit processing. Blanching reduces enzyme activity and improves color in some cases. Water activity reduction by osmodehydration (OD) with concentrated sugar/salt solutions, at low temperature, allows us to obtain minimally processed fruit or to reduce freezable water in order to improve frozen preservation. In this work, the influence of steam blanching pretreatment of mango (var. Kent) for $1.5 \mathrm{~min}$ on the kinetics of osmotic dehydration and pulsed vacuum osmotic dehydration was analyzed. Treatments were carried out at $30^{\circ} \mathrm{C}$ with 59.3 Brix sucrose solution. At several times of processing (till $5 \mathrm{~h}$ ) samples were analyzed as to weight and volume changes and moisture and soluble solid concentration and water activity. Kinetics of sugar and water net gains were analyzed as well as changes in the fruit liquid phase (FLP: water plus soluble solids) composition. Development of volume changes, density and water activity of the samples in the different treatments was compared. PVOD treatments show faster kinetics in solute and water gains, and slightly higher values of effective diffusivity in the FLP, especially in blanched samples. Volume changes in OD treatments were fully explained in terms of the FLP volume changes. In PVOD treatments, deviations from this behavior were observed due to sample impregnation-deformation during vacuum pulse.

Key Words: Osmostic Dehydration, Blanching, Mango.

\section{INTRODUCCIÓN}

La deshidratación osmótica (OD) es un proceso en creciente evolución en la industria alimentaria, la cual consiste en la inmersión del alimento sólido, ya sea entero o en trozos, en disoluciones acuosas de alta concentración. Su beneficio radica en la disminución en la actividad de agua del producto, conservando sus condiciones organolépticas. Los procesos osmóticos y la impregnación a vacío de frutas han sido estudiados en varios trabajos (Fito et al, 1993; Fito y Chiralt, 1995; Barat et al, 1997; Barat, 1998) donde los procesos han sido llamados PVOD (Deshidratación Osmótica con Pulso de Vacío). Durante la etapa a vacío el gas en los espacios intercelulares se expande y sale parcialmente del sistema y al restituir la presión se promueve la entrada del líquido en los poros del producto. Dicho mecanismo se da de forma muy rápida cuando se restaura la presión atmosférica del sistema, después de un corto período de tratamiento a vacío (Barat, 1998 y Barat et al, 1998). La aplicación del escaldado en las frutas reduce su actividad enzimática y mejora su color en algunos casos mediante la 
inactivación de las enzimas Polyphenoloxidasa (PPO) y Peroxidasa (PDO). El objetivo de este trabajo es analizar la influencia del escaldado sobre la transferencia de masa (agua más solutos) durante el proceso de deshidratación osmótica (DO) y deshidratación osmótica con pulso de vacío (PVOD).

\section{MATERIALES Y MÉTODOS}

Se emplearon muestras cilíndricas de mango var. kent $(1.5 \mathrm{~cm}$ altura y $2 \mathrm{~cm}$ diámetro), la fruta se seleccionó con un similar grado de maduración $(0.152 \pm 0.006$ Brix) y firmeza del fruto. Se usó una disolución de sacarosa de grado alimentario $(59,3$ Brix y aw=0.894) como solución osmótica (5O). Se empleó un equipo piloto de deshidratación osmótica con control de temperatura, presión y recirculación de 50 ( 10 $\mathrm{m}^{3} / \mathrm{h}$ ). Los tratamientos osmóticos se realizaron a $30{ }^{\circ} \mathrm{C}$. Las muestras de mango se sumergieron en la solución osmótica y a diferentes tiempos de tratamiento $(5,10,15$, $30,60,90,120,180$ y $300 \mathrm{~min}$ ) se retiraron de la solución y se analizó su variación de peso, contenido de agua y de solutos. En el tratamiento PVOD, las muestras se sumergieron en la 50 y previamente a la OD se aplicó un pulso de vacío (50 mbar durante 5 minutos). Los tratamientos de escaldado (BM: Blanching Mango) se realizaron con vapor de agua durante 1.5 min previo a DO. Las muestras de mango no escaldadas (MBM: Mon Blanching Mango) se deshidrataron directamente (OD o PVOD).

Se determinó el contenido de agua $\left(x_{w}\right)$, sólidos solubles $\left(x_{s}\right)$, actividad de agua $\left(a_{w}\right)$, volumen, densidad y masa en muestras de mango frescas y tratadas. Los sólidos solubles se determinaron por medio de un refractómetro (Atago, MAR T3, Japón) a $20^{\circ} \mathrm{C}$. La cuantificación del contenido de agua se realizó mediante estufa al vacío a $60^{\circ} \mathrm{C}$ hasta peso constante según (AOAC 20013 método de estandar). La actividad de agua se midió con un Higrómetro Decagon CX-1 a $35^{\circ} \mathrm{C}$. Se empleó un picnómetro con solución isotónica como líquido de referencia (método de desplazamiento de volumen) para la medida de volumen de la muestra.

\section{RESULTADOS Y DISCUSIÓN}

Recientemente se ha propuesto un modelo (Fito y Chiralt, 1997; Barat, et al., 1997) que contempla la cinética de los cambios de composición del producto a lo largo del proceso de OD y PVOD desde dos puntos de vista: por un lado, los cambios de composición de la FLM, relacionada con aspectos de su estabilidad del producto y su contenido de agua congelable, que se analizan mediante un modelo difusional simplificado, y por otro lado la cinética de pérdida de peso, asociada con la pérdida de agua y ganancia de solutos, la cual está relacionada con el rendimiento del proceso, que se han descrito por un modelo empírico en términos de la raíz cuadrada del tiempo (Fito y Chiralt, 1997; Crank, 1975).

La figura 1 muestra la ganancia de soluto y pérdida de agua (DMs y DMw), definido en trabajos previos (Fito y Chiralt, 1997) como una función de la raíz cuadrada del tiempo para los diferentes tratamientos (OD O PVOD para escaldado (BM) o no-escaldado (MBM)).

Con las ecuaciones (1) y (2) (Fito y Chiralt, 1997) se modelaron los puntos experimentales y se observó una buena distribución lineal. En estas ecuaciones las constantes $k_{\text {wo }}$ y $k_{\text {so }}$ están relacionadas con ganancias netas muy rápidas ocurridas en el tejido por acción del mecanismo hidrodinámico (HDM), mientras que las constantes cinéticas $k_{w}$ y $k_{s}$ están relacionadas con las velocidades de transferencia de masa asociadas con el mecanismo difusional y osmótico (Fito y Chiralt, 1997).

$$
\begin{aligned}
& \Delta \mathrm{M}_{\mathrm{w}}=\mathrm{k}_{\mathrm{w}} \mathrm{t}^{0,5}+\mathrm{k}_{\mathrm{w}} \mathrm{O} \\
& \Delta \mathrm{M}_{\mathrm{s}}=\mathrm{k}_{\mathrm{s}} \mathrm{t}^{0,5}+\mathrm{k}_{5} \mathrm{O}
\end{aligned}
$$


Los cambios composicionales de la fase liquida de la fruta $\left(z_{t}^{w}\right)$ para los diferentes tratamientos se analizaron en términos de la fuerza impulsora reducida $(Y=Y w=Y s$ ) definida por la ecuación (3). Para cada tratamiento se consideró como concentración de equilibrio $\left(z_{e}\right.$ ) $)$ la concentración de la solución osmótica (Barat, et al., 1998).

El coeficiente de difusión efectivo $\left(D_{e}\right)$ en la fase líquida de la fruta se obtuvo mediante el ajuste de los datos experimentales $1-Y w$ vs $t^{1 / 2}$ según la ecuación 3. Esta ecuación se obtuvo aplicando la regla de Mewman para un cilindro finito a partir de las ecuaciones Fickianas simplificadas (Crank, J., 1975). De la pendiente de la recta ajustada se calculó $D_{e}$

$$
Y_{w}=\left(Z_{t}^{w}-Z_{e}^{w}\right) /\left(Z_{0}^{w}-Z_{e}^{w}\right)=1-\left(\frac{2}{r}+\frac{4}{l}\right)\left(\frac{D_{e} t}{\Pi}\right)^{1 / 2}
$$

Donde: $l=$ semi-altura del cilindro

$$
r=\text { radio del cilindro }
$$

\begin{tabular}{|l|c|c|c|c|c|c|c|c|}
\hline Treatment & $K_{w 0}$ & $K_{w}$ & $r^{2}$ & $K_{s 0}$ & $K_{s}$ & $r^{2}$ & $\begin{array}{c}D_{e} \cdot 10^{-10} \\
m^{2} / 5\end{array}$ & $r^{2}$ \\
\hline MBM-PVOD & - & -0.316 & 0.920 & - & 0.089 & 0.462 & 1.13 & 0.897 \\
\hline MBM-OD & - & -0.320 & 0.972 & - & 0.054 & 0.452 & 0.77 & 0.970 \\
\hline BM-PVOD & -8.238 & -0.326 & 0.871 & 1.495 & 0.127 & 0.935 & 1.63 & 0.929 \\
\hline BM-OD & -5.871 & -0.289 & 0.917 & 0.069 & 0.080 & 0.665 & 0.92 & 0.950 \\
\hline
\end{tabular}

Tabla 1.Constantes cinéticas para la pérdida de agua $\left(K_{\text {wo }} ; K_{w}\right)$ y ganancia de solutos $\left(K_{\text {so }} ; K_{s}\right)$ y la constante cinética para los cambios composicionales en la fase líquida de la fruta $\left(D_{\mathrm{e}}\right)$.

La Tabla 1 muestra las constantes cinéticas $\left(K_{\text {wo }}\right.$ $\left.K_{w}\right)\left(K_{s o} ; K_{s}\right)$ y $D_{e}$, junto a los valores de $r^{2}$ para cada tratamiento, obtenidos de los valores experimentales mediante el ajuste de las ecuaciones (1), (2) y (3). Se observa que las constantes $k_{\text {wo }}$ y $k_{\text {so }}$ se potencian por efecto del escaldado donde parece producirse una ganancia de sólidos solubles por impregnación a tiempos cortos, probablemente debido a la descompartimentación de la estructura de las células superficiales del tejido de la fruta ocasionado por el tratamiento térmico. Esta apertura de células se traduciría en una ganancia de solutos debido a la pérdida de la selectividad al transporte de la membrana celular. Los tratamientos PVOD muestran valores de $K_{5} K_{w}$ ligeramente más altas que los tratamientos OD especialmente en las muestras escaldadas, esto se explica en términos de la composición de los espacios intercelulares de la muestra, pues cuando los espacios intercelulares están llenos de líquido, la ganancia de azucares puede aumentar debido al mecanismo difusional. Se observa que el efecto del pulso de vacío incrementa los valores de la difusividad defectiva $\left(D_{e}\right)$ en la fase líquida de la fruta, similar al observado en otros trabajos con productos vegetales (Martinez.Monzó et al., 1998; Fito y Chiralt, 2000) y es coherente con el hecho de que la eliminación de la fracción de aire en las muestras en los tratamientos PVOD comporta un incremento de volumen efectivo para la difusión. A su vez, el efecto térmico del escaldado sobre las membranas celulares se ha observado también en trabajos previos que tiene como consecuencia un incremento en la velocidades de transporte de masa durante la deshidratación de los tejidos vegetales (Alvarez et al., 1995).

Después de 5 horas de deshidratación, las muestras alcanzaron un rango de actividad de agua entre 0.957 y 0.963 , valores coherentes si tenemos en cuenta que las pérdidas de agua están alrededor de un 40\%.

Una relación interesante desde el punto de vista práctico es la existente entre la $a_{w}$ y el contenido de sólidos solubles de la fracción líquida (agua más solutos) de las muestras $\left(Z_{s}\right)$. Con la ecuación lineal (4) se pueden predecir los valores de actividad de agua en función del contenido de sólidos solubles de la fase líquida de la fruta (FLM) (Figura 2).

$$
a_{w}=-0.1037 z_{s}+1.0031\left(R^{2}=0.9539\right)
$$


NBM
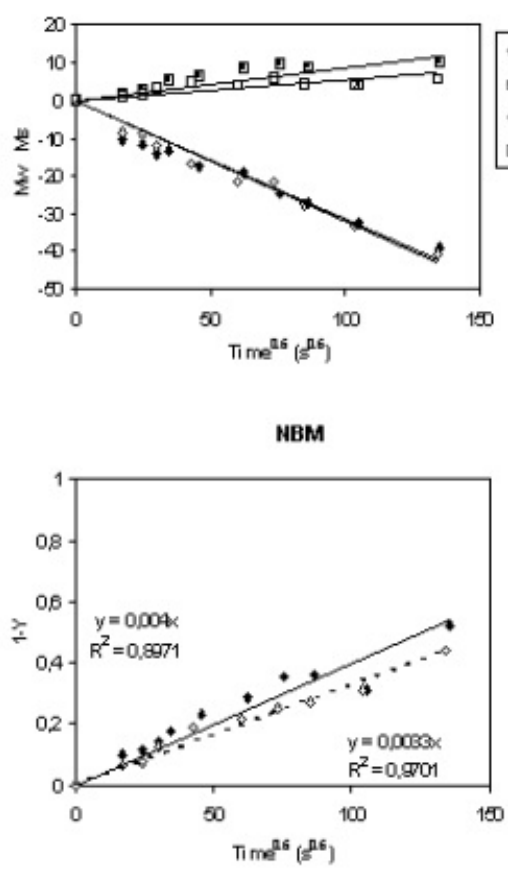

BM
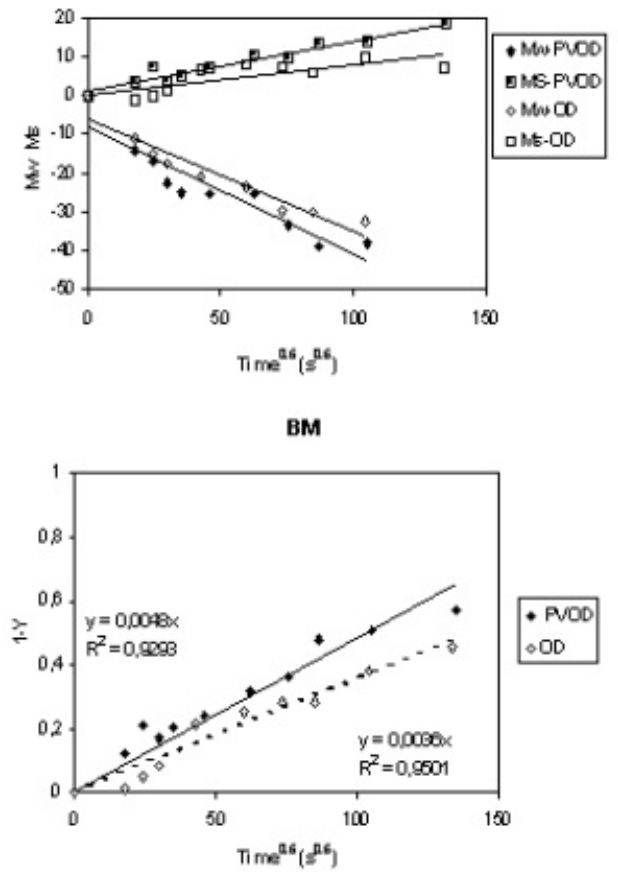

Figura 1Arriba: ganancia de solutos y pérdida de agua en función de la raíz cuadrada del tiempo para muestras no escaldadas (MBM) y escaldas (BM). Abajo: Evolución de la difusividad en la fase líquida de la fruta durante el proceso osmótico

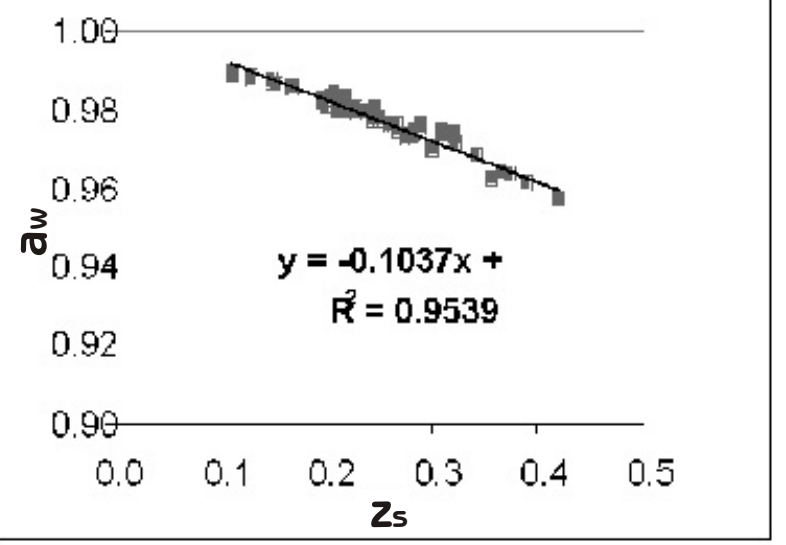

Figura 2. Relación entre $a_{w}$ and $z_{s}$ para los diferentes tratamientos osmóticos

La densidad aparente de las muestras en los tratamientos PVOD siempre es superior a los OD debido a la reducción de la porosidad inicial de las muestras asociada al pulso de vacío, bien por llenado de los poros con 50 o por colapso de los mismos. Para el análisis de los cambios de volumen de la muestra de la fruta durante el proceso de deshidratación osmótica se compararon los cambios de volumen $(\Delta \mathrm{V})$ experimental con el cambio de volumen de la fracción líquida de la muestra $\left(\Delta V_{F L M}\right)$ por unidad de volumen inicial, y para cada tiempo del proceso (Fito et al., 1999). La pérdida de volumen de las muestras se explica principalmente por la pérdida neta de fracción líquida (agua perdida más soluto ganados). La figura 3 muestra esta comparación para muestras no escaldadas y escaldas tanto a OD y PVOD. Se observa que los tratamientos PVOD comportan una desviación de variación de volumen frente a OD, debido por un lado a la impregnación de los poros con líquido externo que representará un aumento en la fracción líquida de la muestra, Por otro lado, la compresión, que supone la restitución de la presión atmosférica después del pulso, implicará 
NBM

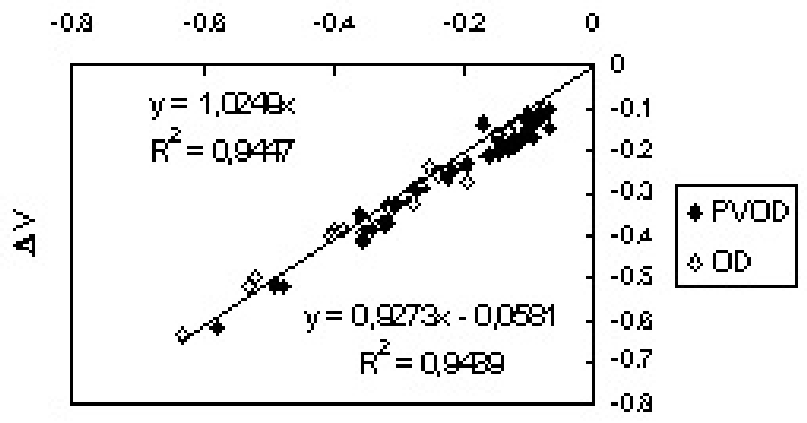

AVFut
BM

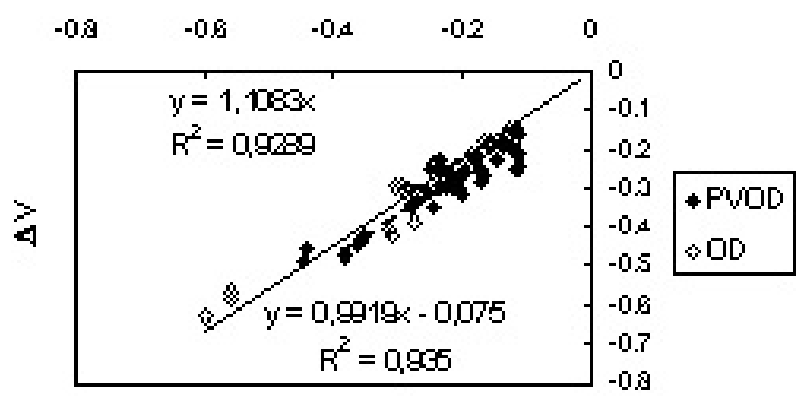

A V Fur

Figura 3. Relación entre el cambio de volumen total $V$ y el cambio de volumen de la fase liquida de la muestra $V_{\text {FLM }}$ para los diferentes tratamientos osmóticos

un colapso parcial del volumen de los poros (deformación) con su correspondiente repercusión en el volumen de la muestra.

\section{CONCLUSIONES}

Los tratamientos PVOD comportaron cinéticas más rápidas en la ganancia de solutos y pérdidas de agua y valores más altos de difusividad efectiva en la fase liquida de la muestra, especialmente en las muestras escaldadas. Los tratamientos PVOD incrementaron la densidad aparente de la muestra, pero no se observa influencia del escaldado sobre los cambios de volumen. En los tratamientos OD los cambios de volumen fueron explicados en términos de la FLM. Los tratamientos PVOD comportan una desviación de variación de volumen frente a OD debido a la impregnación deformación durante el pulso de vacío.

\section{BIBLIOGRAFÍA}

1. ALVAREZ, C.A.; AGUIRRE, R.; GOMEZ, J.; VIDALES, S.; ALZAMORA, S.M.; GERSCHENSOM L.M. (1995). Air dehydration of strawberries: effets of blanching and osmotic pretreaments on the kinnectis of moisture transport. Journal of Food Engineering, 25:167-178.
2.FITO, P., AMDRÉS, A., PASTOR, R., CHIRALT, A., (1993). "Vacuum Osmotic Dehydration of Fruits, in Process Optimization and Minimal Processing of Foods", Singh, P., Oliveira, F. Eds., CRC Press, Boca Ratón, Pp. 107-121.

3.FITO, P., CHIRALT, A., 1995. "An Update on Vacuum Osmotic Dehydration in Food Preservation by Moisture Control: Fundamentals and Applications", G.V. BarbosaCánovas, J. Welti-Chaves Eds., Technomic Pub.Co., Inc. Lancaster, pp. 351-372.

4. FITO, P.; AMDRÉS, A.; CHIRALT, A. and PARDO, P. (1996). Coupling of hydrodynamic mechanism and deformation-relaxation phenomena during vacuum treatments in solid porous food-liquid systems. J. Food Eng., 27: 229-240.

5. BARAT, J. M., ALVARRUIZ, A., CHIRALT, A., FITO, P., (1997). "A mass transfer modelling in osmotic dehydration", in Engineering and Food at ICEF 7, Jowitt, R., Ed., Sheffield Academic Press, pp. G:81-84.

6. BARAT, J.M., 1998. "Desarrollo de un modelo de la deshidratación osmótica como operación básica". Ph.D. Thesis, UPV, España 
7. BARAT, J.M., CHIRALT, A. AMD FITO, P., (1998). "Equilibrium in cellular food osmotic solution systems as related to structure". Journal of Food Science, 63: 1-5.

8. FITO, P., CHIRALT, A., (1997). "Osmotic Dehydration: An approach of the modelling of solid food-liquid operations", Ch. 13 in Food Engineering 2000, P. Fito, E. OrtegaRodriguez, G.V. Barbosa-Canovas Eds., Chapman and Hall, Mew York, Pp. 231-252.

9. FITO, P.; CHIRALT, A. (2000). Vacuum impregnation of plant tissues. En: Minimal processed fruits and Vegetables. Ed. Alzamora, S.M.; Tapia, M.S.; López-Malo, A Aspen Publishers, Inc. Maryland, 189-201.
10.CRAMK, J., (1975). The mathematics of Diffusion, Clarendon Press, Oxford.

11.MARTÍMEZ-MOMZÓ, J., M. MARTÍMEZMAVARRETE, P. FITO, AMD A. CHIRALT. 1998. "Mechanical and Structural Changes in Apple (var. Granny Smith) Due to Vacuum Impregnation with Cryoprotectants". J Food Sci. 63:499-503.

12.FITO, P., CHIRALT, A., MARTÍMEZ-MOMZÓ J. AMD J.M. BARAT. (1999). "Mass Transport and Deformation Relaxation Phenomena in Plant Tissues," oral presentation at ICEF 2000, Welti, J.; Barbosa-Cánovas, G., Ed., Technomics, (in Press). 